\title{
Wideband characteristics of density tapered array antennas
}

\author{
Nguyen Thanh Binh ${ }^{1}$, Nguyen Quoc Dinh ${ }^{2}$, Yoshihide Yamada ${ }^{3}$ \\ ${ }_{1,2}$ Department of Radio-Electronics Engineering, Le Quy Don Technical University, Vietnam \\ ${ }^{3}$ Malaysia-Japan International Institute of Technology UTM, Malaysia
}

\begin{tabular}{l}
\hline \hline Article Info \\
\hline Article history: \\
Received Mar 11, 2020 \\
Revised Jul 23, 2020 \\
Accepted Sep 7, 2020 \\
\hline
\end{tabular}

Keywords:

Grating lobe

Sidelobe level

Unequal spaced array

Wideband

\begin{abstract}
In this paper, wideband characteristics of density tapered arrays are clarified by comparing directly the array factors and radiation patterns of 3 tapered arrays structures with array factors and radiation patterns of equally spaced arrays. Calculated results for a density tapered distribution array consisting of 30 elements claims that the array can perform within a bandwidth of $2.5: 1$ with grating lobe levels lower than $-7.8 \mathrm{~dB}$. Additionally, this paper shows a method of determining the effectiveness of unequal spacing arrays in the design of actual antennas. This method is based on the calculation and analysis of input impedance of array elements caused by mutual coupling effects among array elements.
\end{abstract}

This is an open access article under the CC BY-SA license.

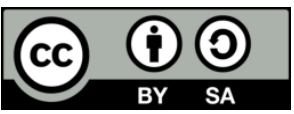

\section{Corresponding Author:}

Nguyen Quoc Dinh

Department of Radio-Electronics Engineering

Le Quy Don Technical University

236 Hoang Quoc Viet Road, Bac Tu Liem District, Hanoi, Vietnam

Email: dinhnq@mta.edu.vn

\section{INTRODUCTION}

Currently, next generation mobile communication system (5G) is developing $[1,2]$. For mobile base station antennas, multi beam and wideband characteristics are requested in addition to low sidelobe characteristics of present base station antennas [3, 4]. In a present mobile base station antenna, an equally spaced linear array configuration is employed as shown in Figure 1(a). Low sidelobe characteristics are achieved by giving adequate excitation coefficients (amplitude and phase) to array elements [5-7], and/or changing the radiation characteristics of elements in the array [8, 9]. In case of providing excitation coefficients to all elements, feeding network includes multiple power dividers and feeder lines that have different values [10-12]. Herein, phase values determined by feeder line lengths, which tend to reduce array antenna's bandwidth. In addition, the appearance of grating lobes in array factors (AF) [13] when equally spaced arrays (ESA) performing within wideband frequencies makes it more difficult in manufacturing actual antenna models. For the purpose of increasing operation frequency range, the density tapered array (DTA) configuration [14, 15] shown in Figure 1(b) will be promising. The excitation coefficients for all array elements are uniform. Here, the feed line lengths from the input to all array elements are equal. Then the feed network has frequency independent characteristics. The DTA antenna will be expected to achieve low sidelobe characteristics in a wide range of frequency.

For previous researches on unequally spacing or density tapered arrays, some method of designing unequally spaced arrays was proposed in [16-22] which achieved side-lobes level (SLL) lower than $-25 \mathrm{~dB}$. In $[23,24]$, the particle swarm optimization algorithm is applied to determine the allocation of elements to achieve the minimum sidelobe level and null control in the array radiation pattern. The main drawbacks of 
these methods are that the spacing between two consecutive elements at the array center was lower than a half of wavelength and/or it required excitation coefficients to array elements with various values. In [25, 26], the evolution algorithm was used to optimize element allocations of 32-element arrays, which resulted in the SLLs around $-22.5 \mathrm{~dB}$. Nevertheless, the wideband characteristic of those structures was not clarified. The early studies about the wideband characteristic of unequally spaced arrays were shown in [27-29], and some experiments were reported in [30-34]. Evidence in the method of designing unequal spacing arrays has been stated in [35]. However, the effect of elements radiation, the mutual coupling effect and the effect of changing the density of them to total radiation pattern of the array were not clarified. Thus, more detailed research on tapered distribution arrays is necessary.

In this paper, wideband characteristics of tapered distribution arrays will be shown by considering array factors and radiation pattern of an array consisting of 30 elements that has operating bandwidth of 2.5:1. In section 2, design, array factors calculation method and radiation pattern of 3 DTAs will be discussed. Numerical results based on Method of Moment will be shown in Section 3. Additionally, radiation effects of array elements and mutual effects among elements to the overall radiation pattern of the array will also be clarified.

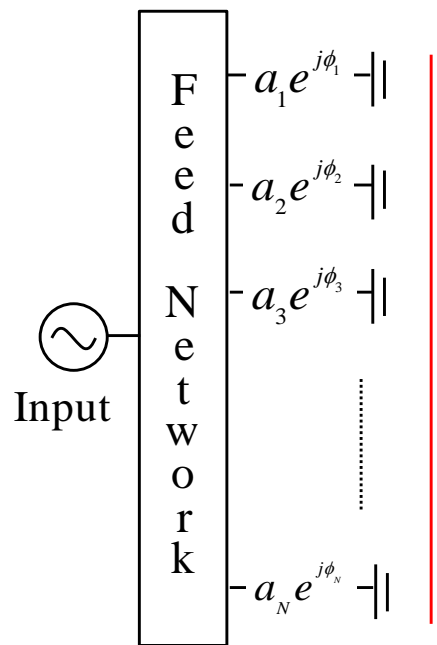

(a)

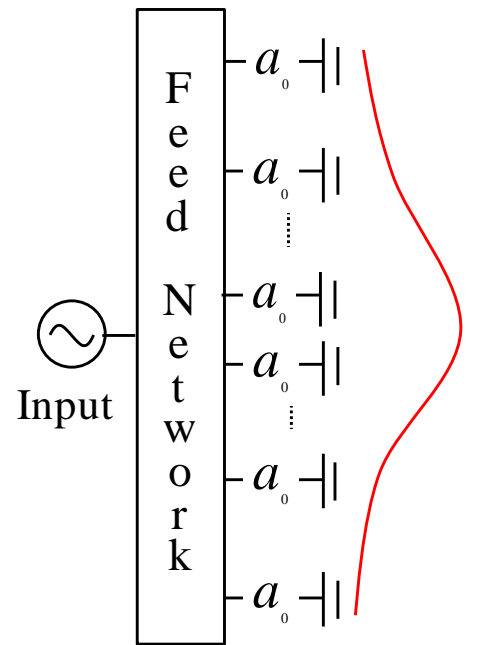

(b)

Figure 1. Mobile base station antenna configurations, (a) present antenna, (b) density tapered array

\section{METHOD TO ACHIEVE TAPERED LINEAR ARRAY}

\subsection{Allocations of array elements}

Figure 2 shows a linear antenna array consisting of $2 \mathrm{~N}$ elements which are symmetrically allocated along z-axis. The spacing between two consecutive elements is denoted as $d_{i}(i=1 \sim N-1)$. The total length of the array is denoted as $L . L$ is fixed and determined by (1).

$$
L=0.7 \times(2 N-1) \lambda_{1}
$$

where, $\lambda_{1}$ is wave-length at lowest frequency.

To achieve tapered distribution, $d_{i}$ gaps among array elements is given by

$$
d_{i}=d_{c}+i \times \Delta d \quad(i=1,2, \ldots, N-1) .
$$

where, $d_{c}$ is the spacing between two elements at the array center, and $\Delta d$ expresses the degree of unequally spacing. When $\Delta d=0$, the array is allocated equally. When the number of array elements is fixed at $2 N=30$, the total length $L$ of the array determined by (1) and (2) is as followed

$$
L=\left(29 d_{c}+210 \Delta d\right) \lambda_{1}
$$


When $d_{c}$ obtains the values of $0.7 \lambda_{1}, 0.6 \lambda_{1}, 0.5 \lambda_{1}$ and $0.4 \lambda_{1}$, the value of $\Delta d$ is given by (3) and shown in Table 1. Herein, the equal spacing array is labeled as ESA and density tapered arrays are labeled as DTA1, DTA2 and DTA3 corresponding with $d_{c}=0.6 \lambda_{1}, 0.5 \lambda_{1}$ and $0.4 \lambda_{1}$.

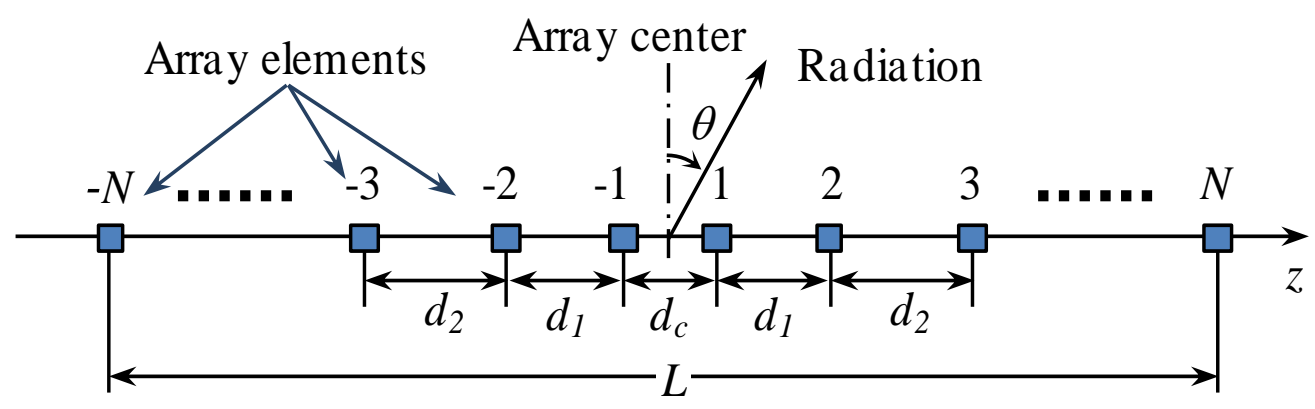

Figure 2. Geometry of a linear symmetrical array

Table 1. Array element spacing (normalize by $\lambda_{1}$ )

\begin{tabular}{ccccc}
\hline & Equal & \multicolumn{3}{c}{ Density tapered } \\
& ESA & DTA1 & DTA2 & DTA3 \\
\hline$d_{c}$ & 0.7 & 0.6 & 0.5 & 0.4 \\
$\Delta d$ & 0 & 0.01381 & 0.02762 & 0.04143 \\
\hline
\end{tabular}

\subsection{Radiation pattern}

Radiation pattern of array in Figure 1 given by the following equation [13]

$$
\bar{E}(\theta)=\bar{E}_{\text {ele }}(\theta) \times A F(\theta)
$$

where

$$
A F(\theta)=\sum_{n=-N, n \neq 0}^{N} I_{n} e^{j\left(k s_{n} \sin \theta+\beta_{n}\right)}
$$

is the array factor, and $E_{\text {ele }}(\theta)$ is the elemental radiation. $I_{n}$ and $\beta_{n}$ denote excitation current and excitation phasor of $n^{\text {th }}$ element. $S_{n}$ is the location of $n^{\text {th }}$ element. To simplify the feeding network and ensure that its operation is independent of frequencies, excitation phasor and excitation amplitude to all of the elements will be maintained unchanged. Hence (5) is rewritten as

$$
A F(\theta)=\sum_{n=-N, n \neq 0}^{N} I_{0} e^{j k s_{n} \sin \theta}
$$

where, $I_{0}$ is excitation current of each elements in an isolated system.

\section{NUMERICAL RESULTS AND ANALYSIS}

In this section, to evaluate wideband characteristics of the array structure shown in subsection 2.1, array factors and radiation pattern of DTAs will be directly compared with array factors and radiation pattern of the ESA. Four different frequencies $\left(f_{1}, f_{2}=1.5 f_{1}, f_{3}=2 f_{1}\right.$ and $\left.f_{4}=2.5 f_{1}\right)$ corresponding with bandwidth $f_{4}: f_{1}$ of 2.5:1 is chosen for calculation. Note that when calculating frequencies change, the locations of elements remain unchanged. In other words, the total length of the array is fixed according to the wavelength $\lambda_{1}$ when the operating frequencies of the array change. Herein, $\lambda_{1}$ is the corresponding wavelength at the lowest frequency $\left(f_{1}\right)$. 


\subsection{Array factor}

\subsubsection{Equally spaced array $\left(d_{c}=0.7 \lambda_{1}\right)$}

Frequency responses of equally spaced arrays are shown in Figure 3. Obviously, grating lobes are produced at high frequencies. The angle $\left(\theta_{G}\right)$ of grating lobe position is given by the expression

$$
\sin \theta_{G}=\frac{\lambda_{i}}{0.7 \lambda_{1}} \quad(i=2,3,4) .
$$

at $f_{2}, f_{3}$ and $f_{4}, \theta_{G}$ becomes 72.24 degrees, 45.58 degrees and 34.85 degrees, respectively.

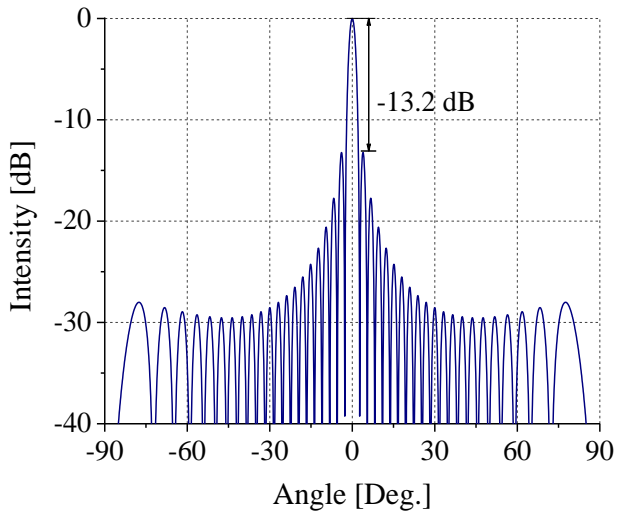

(a)

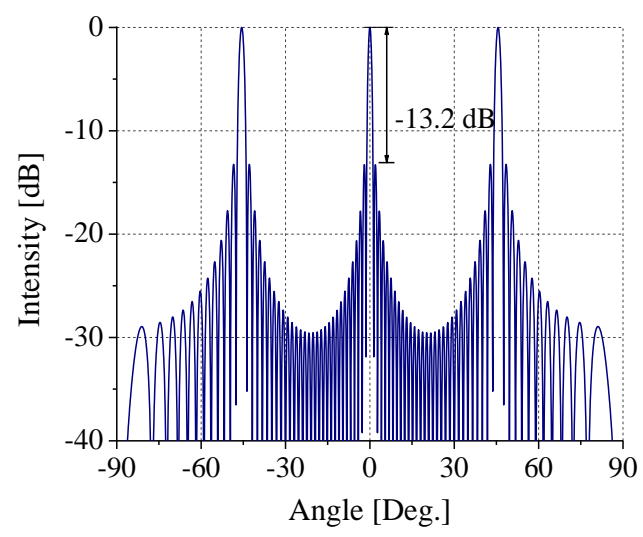

(c)

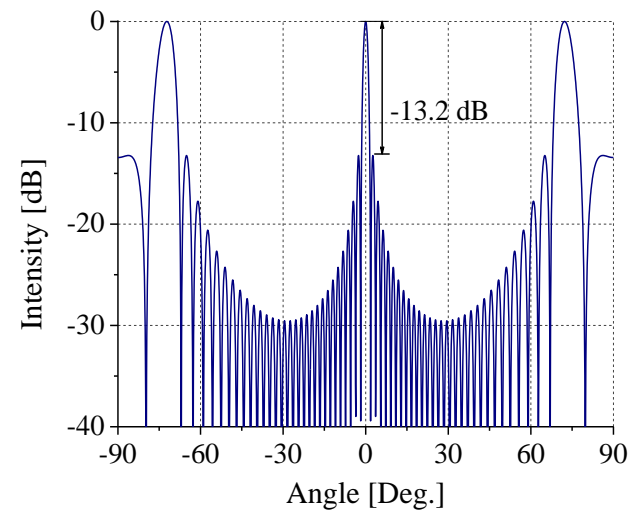

(b)

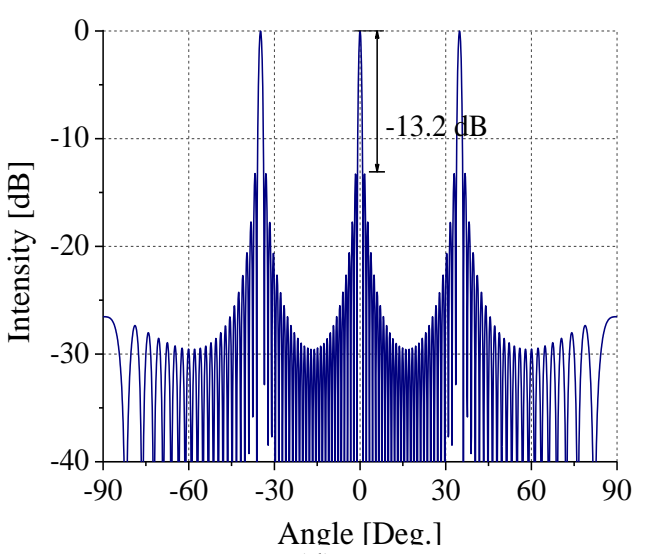

(d)

Figure 3. Array factor of equally spaced array, (a) $f_{1}$, (b) $f_{2}$, (c) $f_{3}$, (d) $f_{4}$

\subsubsection{Density tapered array}

Frequency responses of density tapered array configurations with $d_{c}=0.6 \lambda_{1}$ (DTA1) are shown in Figure 4. Typically, the first sidelobe level (FSLL) of DTA1 reduces $2.4 \mathrm{~dB}$ compared to that of ESA. Interestingly, FSLL remains unchanged at $-15.6 \mathrm{~dB}$ at all frequencies. The next effect is that the grating lobe levels are stretched and those peaks decrease $5 \mathrm{~dB}$ compared to those in ESA. Moreover, the grating lobe peaks remain unchanged at all frequencies.

For the DTA of $d_{c}=0.5 \lambda_{1}$, the array factors are shown in Figure 5. The FSLL and the maximum grating lobe level keep reducing compared to those in Figure 4. Herein, the FSLL and maximum level of grating lobe are $-19.1 \mathrm{~dB}$ and $-7.8 \mathrm{~dB}$, respectively. It can be seen that grating lobe variation is becoming larger.

For the DTA of $d_{c}=0.4 \lambda_{1}$, the array factors are shown in Figure 6. The FSLL keeps decreasing, reaching $-25.4 \mathrm{~dB}$. In contrast, the second sidelobe level increases compared to that in Figure 5, reaching $-17.2 \mathrm{~dB}$. The reason for this increment is that the spacing among elements at the array edge become too large. 


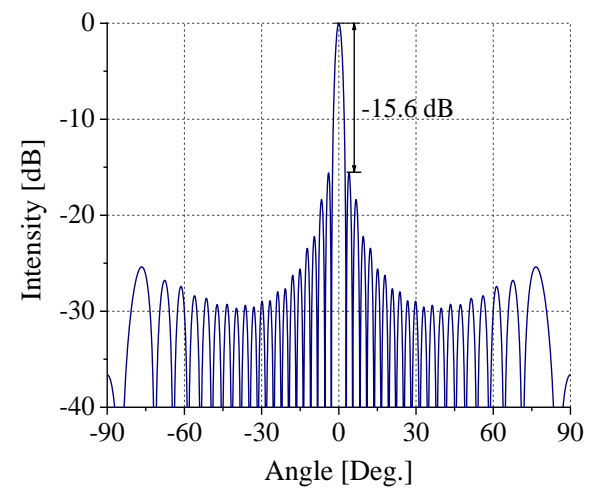

(a)

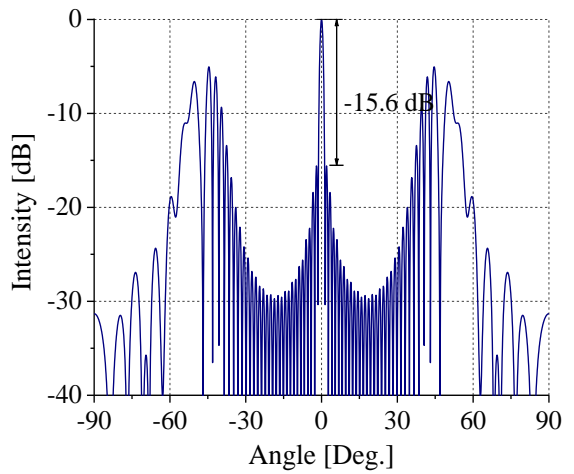

(c)

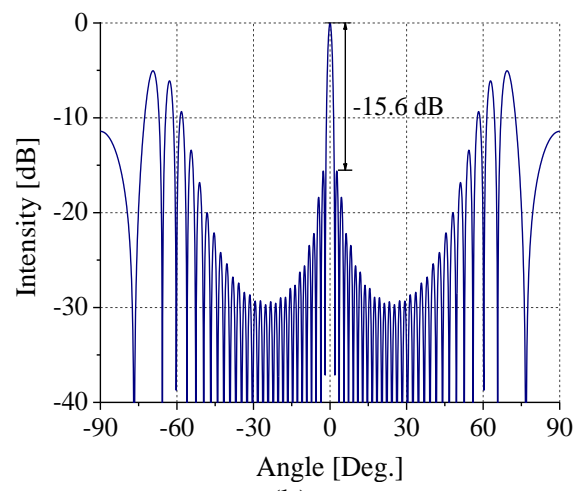

(b)

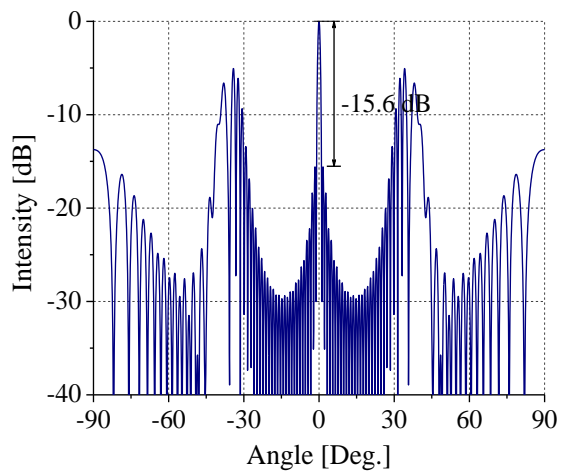

(d)

Figure 4. Array factor of density tapered array (DTA1), (a) $f_{1}$, (b) $f_{2}$, (c) $f_{3}$, (d) $f_{4}$

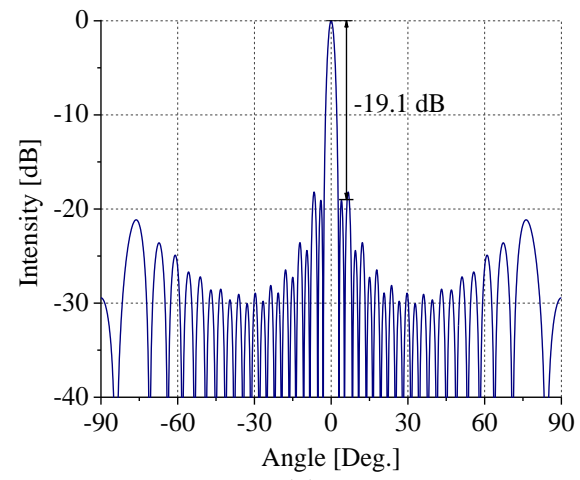

(a)

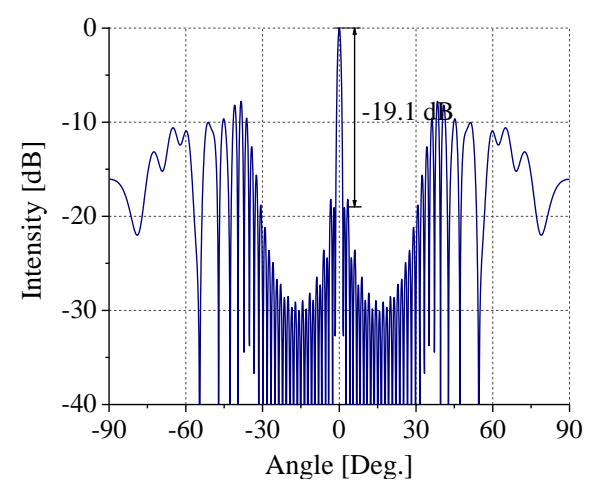

(c)

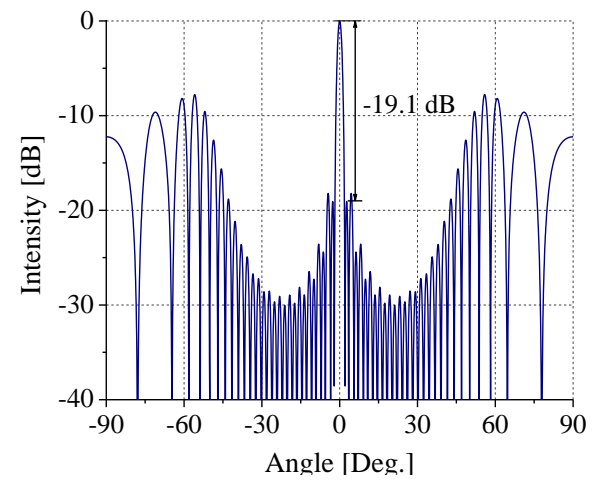

(b)

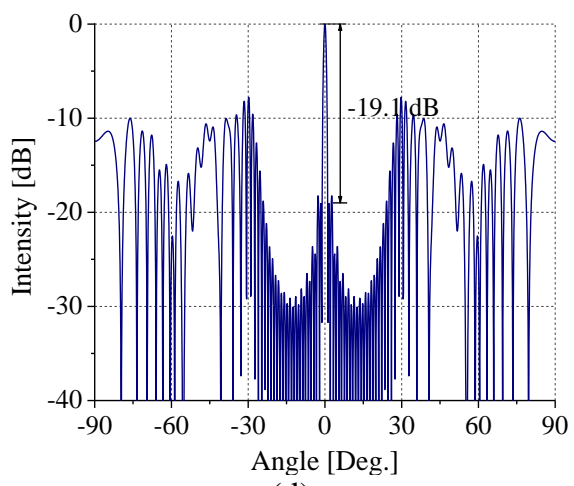

(d)

Figure 5. Array factor of density tapered array (DTA2), (a) $f_{1}$, (b) $f_{2}$, (c) $f_{3}$, (d) $f_{4}$ 


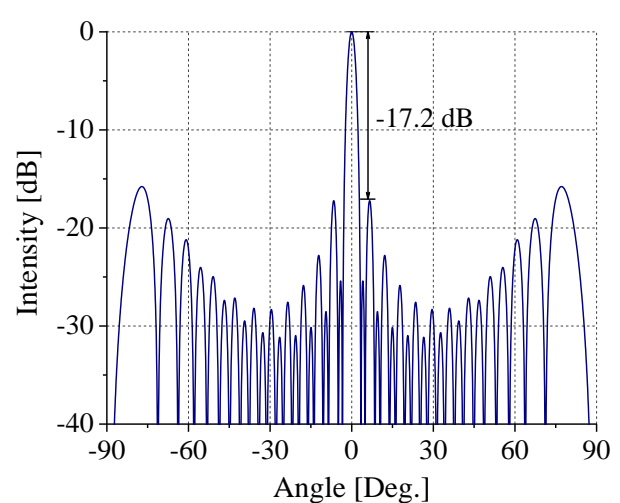

(a)

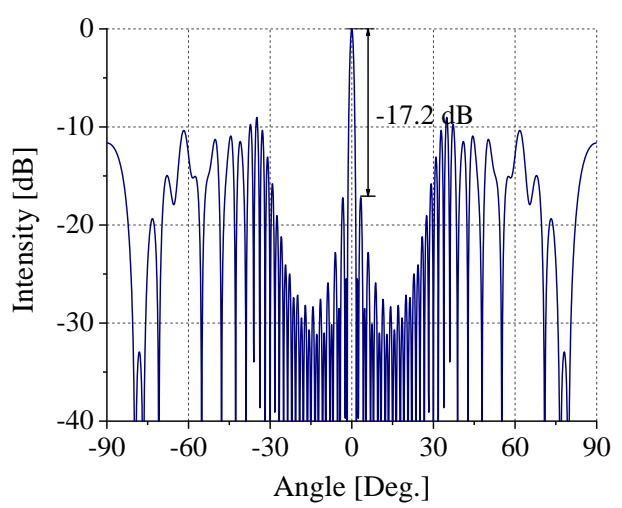

(c)

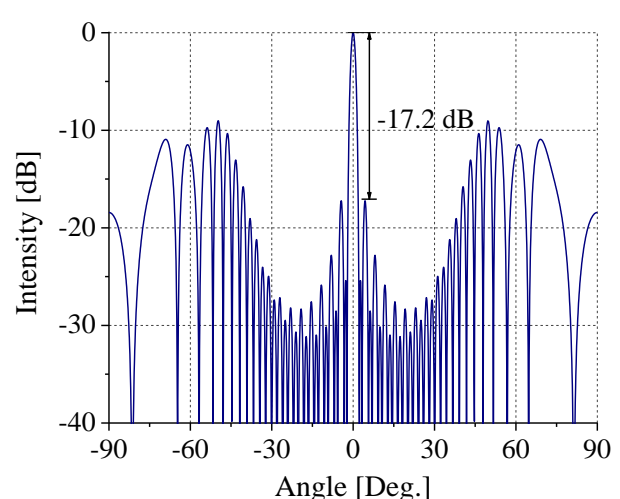

(b)

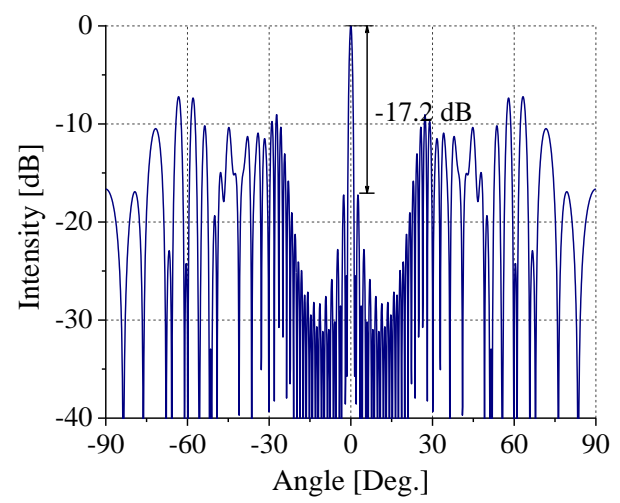

(d)

Figure 6. Array factor of density tapered array (DTA3), (a) $f_{1}$, (b) $f_{2}$, (c) $f_{3}$, (d) $f_{4}$

\subsubsection{Summary}

To summary the features of array factors, the FSLL and grating lobe levels are chosen and shown in Figure 7. The FSLL characteristic is shown in Figure 7(a), in which the FSLL descreases gradually when the density of elements at the array center increases. Moreover, those values remain unchanged at all frequencies. For grating lobe levels, the maximum levels of grating lobe are likely to decrease when the density of elements at the array center increases. However, at $d_{c}=0.4 \lambda_{1}$ and $f_{4}=2.5 f_{1}$ the grating lobe peaks tend to increase because the spacings between elements at the edge of the array become too large.

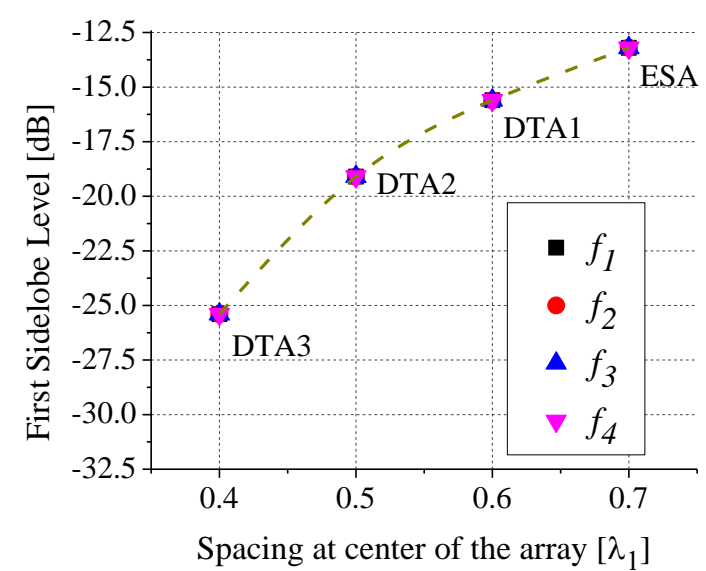

(a)

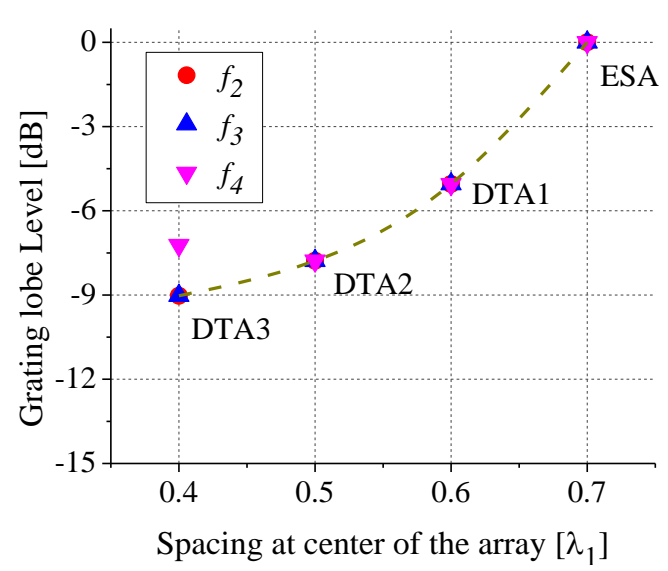

(b)

Figure 7. Comparison of FSLL and grating lobe level in terms of spacing at the array center 


\subsection{Dipole elements}

To consider effects of array elements to its overall radiation pattern, dipole antennas with the length of $0.48 \lambda_{i}$ and the diameter of $10^{-3} \lambda_{i}$ are selected, where $\lambda_{i}(i=1,2,3,4)$ are the wavelengths of corresponding frequencies $f_{1}, f_{2}, f_{3}$ and $f_{4}$. Herein, typical examples at $f_{1}$ and $f_{3}$ are selected.

\subsubsection{Equally spaced array}

Radiation pattern of ESA is shown in Figure 8. Obviously, the FSLL in Figure 8 is kept unchanged and the FSLL values in this case are equivalent to the results in Figure 3. Sidelobes which are far from the main lobe tend to decrease because of radiation characteristics of array elements. As a result, the maximum level of grating lobe is likely to decrease.

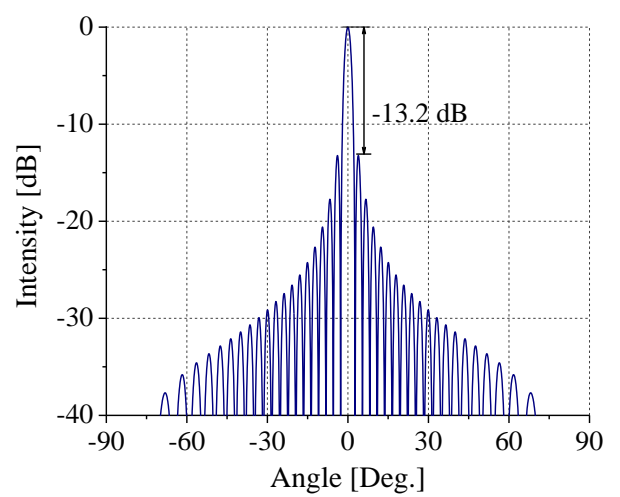

(a)

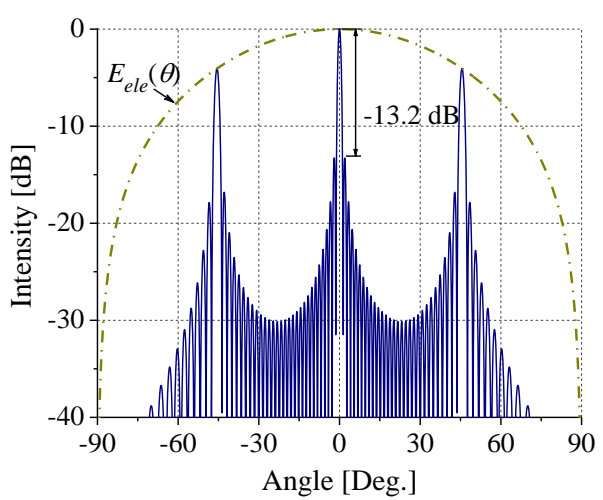

(b)

Figure 8 . Radiation pattern of equally spaced array, (a) $f_{1}$, (b) $f_{2}$

The input impedances of elements in ESA are shown in Figure 9. Clearly, the input resistance (Rm) and reactance $(\mathrm{Xm})$ components are approximately uniform among array elements. When the input impedance is uniform, the amplitude and phasor of each element are similar to the others, which makes it convenient to determine excitation in terms of amplitude and phasor to array elements.

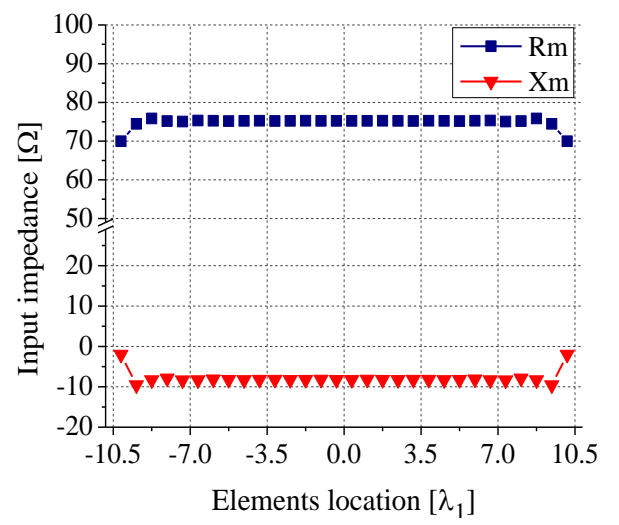

(a)

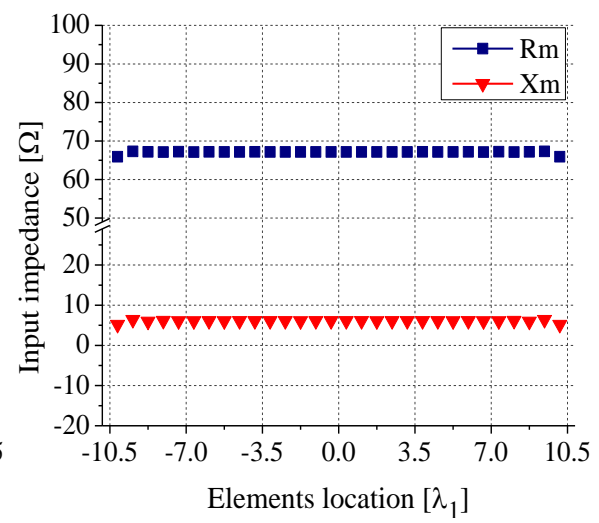

(b)

Figure 9. The input impedances of half-wavelength elements in equally spaced array, (a) $f_{1}$, (b) $f_{2}$

\subsubsection{Density tapered array}

Radiation pattern of DTA when $d_{c}=0.5 \lambda_{1}$ is shown in Figure 10. The FSLLs are much more different compared to those in Figure 5. In particular, at the frequency of $f_{1}$, the FSLL in Figure 10(a) increases $5.1 \mathrm{~dB}$ compared to that of Figure 5(a). The reason for this discrepancy can be explained through results shown in Figure 11(a). Accordingly, the intense mutual coupling effects among elements at the array 
center cause the increase of input impedance of elements at the array center. Since the input impedance of array center elements increases, the excitation current at the array center elements decreases. This results in the increase in the FSLL. For sidelobes far from the main lobe, the maximum levels of those lobes tend to decrease due to the radiation characteristic of array elements. At the frequency of $f_{3}$ shown in Figure 10(b), the FSLL is $2.2 \mathrm{~dB}$ lower than that in Figure 5(c), which can be explained by results in Figure 11(b). Herein, the input impedance of elements at array center is lower than those at array edge. This result makes the current distribution at the array center higher than that at the array edge. This feature makes the FSLL in Figure 10(b) lower than that in Figure 5(c). For grating lobe levels, the peak levels decrease significantly due to the radiation characteristic of array elements.

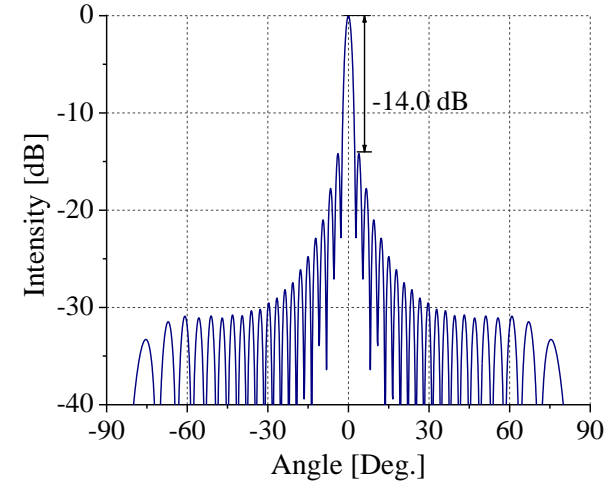

(a)

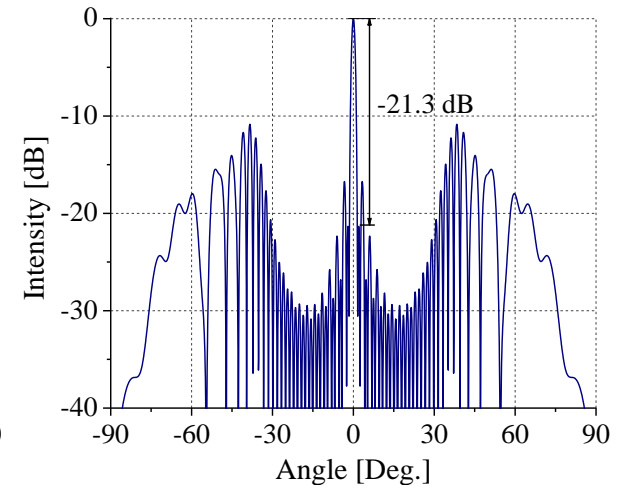

(b)

Figure 10. Radiation pattern of density tapered array (DTA2), (a) $f_{1}$, (b) $f_{2}$

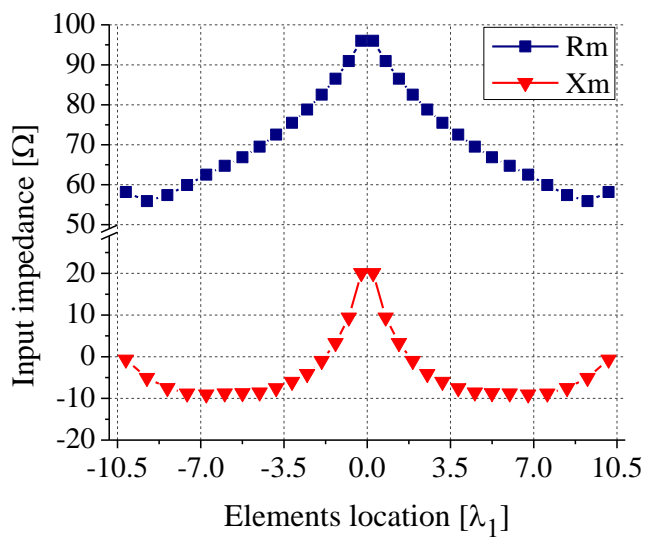

(a)

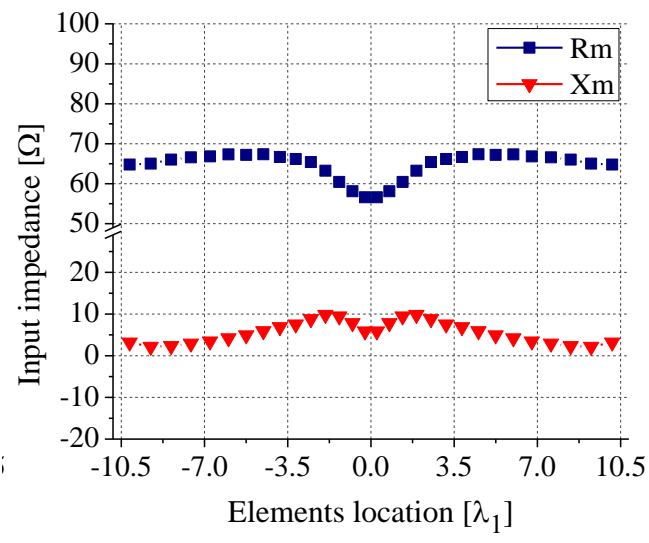

(b)

Figure 11. The input impedances of half-wavelength elements in density tapered array (DTA2), (a) $f_{1}$, (b) $f_{2}$

\subsubsection{Summary}

The typical characteristics of DTA including FSLL and grating lobe levels are shown in Figure 12. As can be seen from Figure 12(a), the FSLL of DTA structure is equivalent to that in ESA at the frequency of $f_{1}$. This is because of the intense mutual coupling effects of elements at the array center. At high frequencies, the mutual coupling effect decreases which results in the reduction of FSLL. Those results show that, the mutual effects among array elements have to be taken into account in calculation and design of tapered arrays. Hence, the techniques of reducing mutual effects among array elements should be considered in unequally spaced array antenna designs. The variation trend of grating lobe peaks in Figure 12(b) is the same to that in Figure 7(b). The different results in Figure 12(b) and Figure 7(b) are caused by radiation characteristics of array elements which make the grating lobe levels in Figure 12(b) lower than those in Figure 7(b). Again, those results claim the ability of compressing grating lobe in a wide frequency range of DTA structure. 


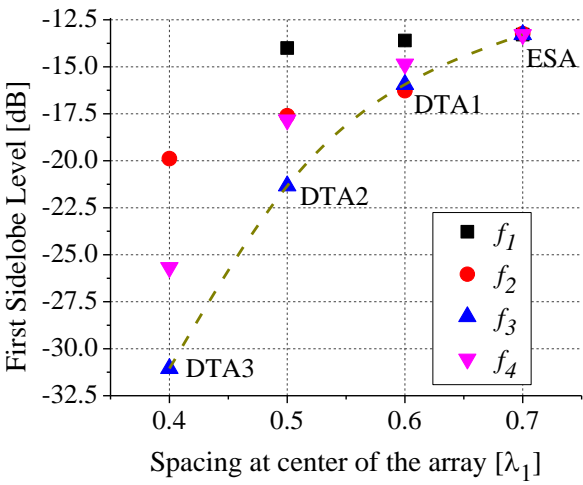

(a)

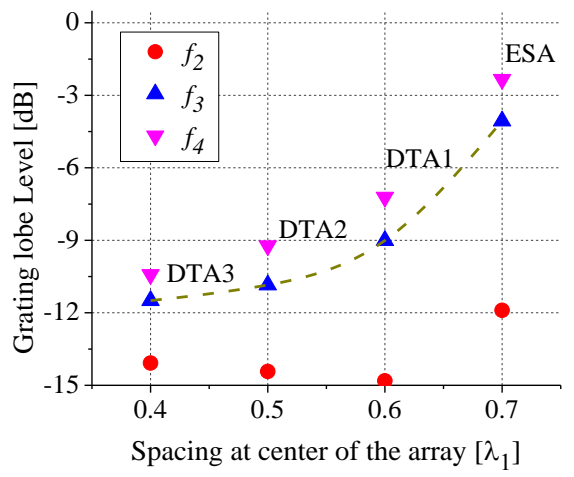

(b)

Figure 12. Comparison FSLL and grating lobe level in terms of spacing at the array center, (a) $f_{1}$, (b) $f_{2}$

\section{CONCLUSION}

In this paper, the authors have proposed a method to examine the effectiveness of density tapered array, which is based on determining input impedance of array elements. In calculations, arrays consisting of 30 elements with various distributions were chosen. The results show that when there is no mutual coupling effect, DTA structures achieved sidelobe levels lower than ESA as well as were able to compress grating lobes within frequency range of 2.5:1. When mutual coupling effect between elements is considered, the wideband characteristic of DTA structure is ensured. Furthermore, the mutual coupling effect is likely to enhance or lower the sidelobe levels which are near the main lobe. This feature shows that mutual coupling effect has to be considered while designing density tapered array antenna.

\section{ACKNOWLEDGEMENTS}

This research is funded by Vietnam National Foundation for Science and Technology Development (NAFOSTED) under grant number 102.04-2018.08.

\section{REFERENCES}

[1] T. S. Rappaport, et al., "Millimeter wave mobile communications for 5G cellular: It Will Work!," IEEE Access, vol. 1, pp. 335-349, 2013.

[2] C. X. Wang, et al., "Cellular architecture and key technologies for 5G wireless communication networks," IEEE Communications Magazine, vol. 52, pp. 122-130, 2014.

[3] Y. Rahayu, et al., "High gain 5G MIMO antenna for mobile base station," International Journal of Electrical and Computer Engineering (IJECE), vol. 9, no. 1, pp. 468-476, 2019.

[4] W. Roh, et al., "Millimeter-wave beamforming as an enabling technology for 5G cellular communications: Theoretical feasibility and prototype results," IEEE communications magazine, vol. 52, pp. 106-113, 2014.

[5] A. A. Amaireh, et al., "The optimal synthesis of scanned linear antenna arrays," International Journal of Electrical and Computer Engineering (IJECE), vol. 10, no. 2, pp. 1477-1484, 2020.

[6] C. L. Dolph, "A current distribution for broadside arrays which optimizes the relationship between beam width and side-lobe level," Proceedings of the IRE, vol. 34, pp. 335-348, 1946.

[7] S. A. Schelkunoff, "A mathematical theory of linear arrays," The Bell System Technical Journal, vol. 22, pp. 80-107, 1943.

[8] M. K. Abdulhameed, et al., "Side lobe reduction in array antenna by using novel design of EBG," International Journal of Electrical and Computer Engineering (IJECE), vol. 10, no. 1, pp. 308-315, 2020.

[9] F. J. Pompei and S. C. Wooh, "Phased array element shapes for suppressing grating lobes," The Journal of the Acoustical Society of America, vol. 111, pp. 2040-2048, 2002.

[10] W. R. Li, et al., "Switched-beam antenna based on modified Butler matrix with low sidelobe level," Electronics Letters, vol. 40, pp. 290-292, 2004.

[11] T. T. Tang, et al., "A Feeding Network with Chebyshev Distribution for Designing Low Side-lobe Level Antenna Arrays," VNU Journal of Science: Computer Science and Communication Engineering, vol. 33, pp. 16-21, 2017.

[12] K. Wincza, et al., "Reduced sidelobe four-beam antenna array fed by modified Butler matrix," Electronics Letters, vol. 42, pp. 508-509, 2006.

[13] A. B. Constantine, "Antenna theory: analysis and design," 4th ed. Hoboken, New Jersey, John Wiley \& Sons, 2016.

[14] T. A. Milligan, "Modern antenna design," John Wiley \& Sons, 2005.

[15] R. E. Collin and F. J. Zucker, “Antenna theory: part 1,” New York, McGraw-Hill, 1969. 
[16] O. M. Bucci, et al., "Deterministic synthesis of uniform amplitude sparse arrays via new density taper techniques," IEEE Transactions on Antennas and Propagation, vol. 58, pp. 1949-1958, 2010.

[17] A. Ishimaru, "Theory of unequally-spaced arrays," IRE Transactions on Antennas and Propagation, vol. 10, pp. 691-702, 1962.

[18] A. Ishimaru, "Unequally Spaced Arrays Based on the Poisson Sum Formula," IEEE Transactions on Antennas and Propagation, vol. 62, pp. 1549-1554, 2014.

[19] W. Sandrin, et al., "Design of arrays with unequal spacing and partially uniform amplitude taper," IEEE Transactions on Antennas and Propagation, vol. 17, pp. 642-644, 1969.

[20] H. Schuman and B. Strait, "On the design of unequally spaced arrays with nearly equal sidelobes," IEEE Transactions on Antennas and Propagation, vol. 16, pp. 493-494, 1968.

[21] K. K. Yan and Y. Lu, "Sidelobe reduction in array-pattern synthesis using genetic algorithm," IEEE Transactions on Antennas and Propagation, vol. 45, pp. 1117-1122, 1997.

[22] B. Q. You, et al., "Hybrid Approach for the Synthesis of Unequally Spaced Array Antennas With Sidelobes Reduction," IEEE Antennas and Wireless Propagation Letters, vol. 14, pp. 1569-1572, 2015.

[23] N. Jin and Y. Rahmat-Samii, "Advances in particle swarm optimization for antenna designs: real-number, binary, single-objective and multiobjective implementations," IEEE transactions on antennas and propagation, vol. 55, pp. 556-567, 2007.

[24] M. M. Khodier and C. G. Christodoulou, "Linear array geometry synthesis with minimum sidelobe level and null control using particle swarm optimization," IEEE Transactions on antennas and propagation, vol. 53, pp. 2674-2679, 2005.

[25] D. G. Kurup, et al., "Synthesis of uniform amplitude unequally spaced antenna arrays using the differential evolution algorithm," IEEE Transactions on Antennas and Propagation, vol. 51, pp. 2210-2217, 2003.

[26] C. Lin, et al., "Synthesis of Unequally Spaced Antenna Arrays by Using Differential Evolution," IEEE Transactions on Antennas and Propagation, vol. 58, pp. 2553-2561, 2010.

[27] D. King, et al., "Unequally-spaced, broad-band antenna arrays," IRE Transactions on Antennas and Propagation, vol. 8, pp. 380-384, 1960

[28] H. Unz, "Linear Arrays with arbitrarily distributed elements," IRE Transactions on Antennas and Propagation, vol. 8, pp. 222-223, 1960 .

[29] H. Unz, "Nonuniform arrays with spacing larger than one wavelength," IRE Transactions on Antennas and Propagation, vol. AP-10, pp. 647-648, 1962.

[30] N. T. Binh, et al., "Design of density tapered array for arbitrary density distribution," in 2016 International Conference on Advanced Technologies for Communications (ATC), Hanoi, Vietnam, 2016, pp. 375-379.

[31] B. P. Kumar and G. Branner, "Design of unequally spaced arrays for performance improvement," IEEE Transactions on Antennas and Propagation, vol. 47, pp. 511-523, 1999.

[32] C. Song and Q. Wu, "A wide-band phased array antennas with unequal space," in Proceedings of 2012 5th Global Symposium on Millimeter-Waves, 2012, pp. 393-396.

[33] T. K. Tuyen, et al., "Multi-frequency characteristics of unequal element spacing array antenna for mobile base station use," in 2015 International Conference on Advanced Technologies for Communications (ATC), Ho Chi Minh city, Viet Nam, 2015, pp. 540-545.

[34] N. A. Zainal, et al., "Low Sidelobe and Wideband Characteristics of Density Tapered Arrays for 5G Mobile Systems," Jurnal Teknologi, vol. 78, pp. 72-76, 2016.

[35] N. Q. Dinh, et al., "Proof of the density tapering concept of an unequally spaced array by electric field distributions of electromagnetic simulations," Journal of Electromagnetic Waves and Applications, vol. 34, pp. 668-681, 2020.

\section{BIOGRAPHIES OF AUTHORS}
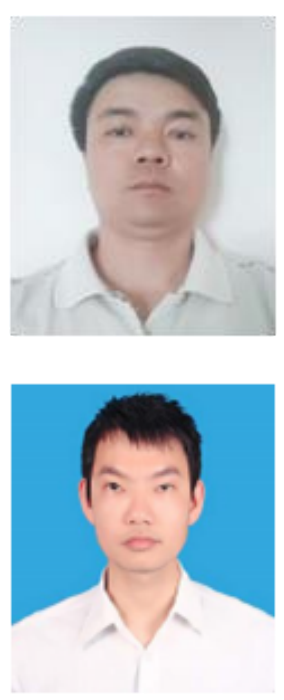

Nguyen Thanh Binh received the B.S. degree from Telecommunication University, Vietnam, in 2008, the M.E. degree from Posts and Telecommunications Institute of Technology, Vietnam, in 2011. He is currently working toward the Ph.D. degree at the Le Quy Don Technical University, Vietnam. His main research interests are array antennas

Nguyen Quoc Dinh is currently an Associate Professor at Faculty of Radio-Electronics Engineering, Le Quy Don Technical University Vietnam. He received the B.E, M.E. and D.E. degrees in Department of Electrical \& Electronic Engineering, National Defense Academy, Yokosuka, Japan, in 2006, 2008, and 2011, respectively. Since 2011, he has been a Research Associate at the Faculty of Radio-Electronics Engineering, Le Quy Don Technical University, Hanoi, Vietnam. His research interests include very small antennas, array antennas, UWB antennas, and MIMO antennas. He is a member of the Institute of Electronics, Information and Communication Engineers (IEICE), Japan. He was the recipient of the Young Scientist Award of the IEICE Antennas and Propagation Society Japan Chapter, Japan (2011). 


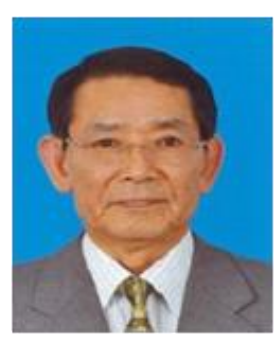

Yoshihide Yamada received the B.E. and M.E. degrees on electronics from Nagoya Institute of Technology, Nagoya, Japan in 1971 and 1973, respectively. And he received the D.E. degree on electrical engineering from Tokyo Institute of Technology, Tokyo, Japan in 1989. In 1973, he joined the Electrical Communication Laboratories of Nippon Telegraph and Telephone Corporation (NTT). Till 1984, he was engaged in research and development of reflector antennas for terrestrial and satellite communications. From 1985, he engaged in R\&D of base station antennas for mobile radio systems. In 1993, he moved to NTT Mobile Communications Network Inc. (NTT DoCoMo). In 1995, he was temporarily transferred to YRP Mobile Telecommunications Key Technology Research Laboratories Co., Ltd. At the same time, he was a guest professor of the cooperative research center of Niigata University, and a lecturer of Science University of Tokyo, both from 1996 to 1997. In 1998, he changed his occupation to a professor of National Defense Academy, Kanagawa, Japan. In 2014, he had started working as a professor at Malaysia-Japan International Institute of Technology, Universiti Teknologi Malaysia, Kuala Lumpur, Malaysia. Now, he is interested in very small antennas, array antennas, aperture antennas and electromagnetic simulation of radar cross section. He received the best paper award and the best tutorial paper award from IEICE in 2013 and 2014, respectively. He is a Fellow member of the IEICE and a member of JSST of Japan. He is also a senior member of IEEE AP society and a member of the ACES. 